\title{
Efecto mediacional del afecto entre las autoevaluaciones centrales y la satisfacción vital en discapacidad intelectual
}

\section{Mediating effects of affect between core self-evaluations and life satisfaction in intellectual disabilities}

\author{
Lourdes Rey Peña y Natalio Extremera Pacheco
}

Universidad de Málaga, España

Disponible online 31 de diciembre de 2016

\begin{abstract}
Este estudio examina el efecto mediacional del afecto en la relación entre las autoevaluaciones centrales (CSE) y la satisfacción vital en personas con discapacidad intelectual. Ciento treinta y cuatro personas con discapacidad intelectual (79 hombres y 55 mujeres) del sur de España completaron la escala de autoevaluaciones centrales (CSES), la escala de afecto positivo y afecto negativo (PANAS), y la escala de satisfacción con la vida (SWLS). La investigación reveló que altos niveles de CSE se asociaban a una mayor satisfacción con la vida. Además, el afecto positivo medió parcialmente la relación entre CSE y satisfacción vital. El modelo final indicó una vía significativa mediante la cual las CSE afectan a la satisfacción vital a través del afecto positivo. Se consideran las limitaciones del estudio y se discuten las implicaciones de los resultados para la mejora de la satisfacción vital en personas con discapacidad intelectual.
\end{abstract}

Palabras clave: Satisfacción con la vida, autoevaluaciones centrales, afecto positivo y negativo, discapacidad intelectual

This study examined the mediating effects of affect on the relationship between core self-evaluations (CSE) and life satisfaction in people with intellectual disabilities. The participants comprised 134 people ( 79 men and 55 women from southern Spain) with intellectual disabilities. All participants completed the core self-evaluations scale (CSES), the positive and negative affect scale (PANAS), and the satisfaction with life scale (SWLS). The results showed that high scores on the CSE were associated with higher life satisfaction. Moreover, positive and negative affect partially mediated the relationship between CSE and life satisfaction. The final model also showed two significant paths from CSE through positive and negative affect to life satisfaction. We present the limitations of the study and discuss the implications of the results for enhancing life satisfaction in people with intellectual disabilities.

Keywords: life satisfacction, core self-evaluations, positive and negative affect, intellectual disability. 


\section{Introducción}

Desde el ámbito de estudio de la psicología positiva ha crecido el interés por conocer cuáles son las variables personales intervinientes o mediadoras que influyen sobre el bienestar personal, y en un sentido más amplio, sobre la calidad de vida (Seligman y Csikszentmihalyi, 2000, Smedema, et al., 2015). En este sentido, el bienestar subjetivo es un área de investigación floreciente dentro del campo de investigación de las experiencias positivas (Danna y Griffin 1999; Diener, Scollon, y Lucas., 2003; Russell 2008). Bajo esta área de estudio han sido objeto de interés constructos tradicionales como la felicidad, la satisfacción con la vida, el afecto positivo y el estado de ánimo (Diener, Suh, Lucas, y Smith, 1997; Kahneman, Diener y Schwarz,1999).

El bienestar subjetivo puede definirse como una combinación de satisfacción vital y el balance de afecto positivo y afecto negativo (Diener, Sandvik, y Pavot, 1991; Larsen Diener, y Emmons, 1985). Más concretamente, la literatura apunta a la existencia de dos componentes principales que configuran el bienestar subjetivo: un componente cognitivo (juicio), que se refiere a la satisfacción global con la vida y a la satisfacción específica en dominios o áreas concretas $\mathrm{y}$, un componente afectivo (emociones), que se divide en afecto positivo o agradable y afecto negativo o desagradable (Diener et al., 1999; Pavot y Diener, 1993). La satisfacción con la vida (componente cognitivo) se refiere, a un juicio mediante el cual el individuo evalúa su calidad de vida en base a los criterios que cada uno elige para evaluarla (Diener et al., 2003; Shin y Johnson, 1978). Una persona con alta satisfacción vital es aquella que compara las circunstancias vitales percibidas por ella misma con un estándar autoimpuesto o una serie de estándares presumiblemente creados y obtiene como resultado una gran coincidencia (Pavot y Diener, 1993). El afecto (componente afectivo) puede entenderse como "las experiencias anímicas y emocionales que experimentamos y que nos informan sobre la marcha de nuestras vidas" (Vázquez y Hervás, 2009, p.23).

El bienestar subjetivo es considerado un componente clave de la calidad de vida de las personas. Históricamente, la satisfacción vital se ha utilizado para examinar la naturaleza subjetiva de la calidad de vida, preguntando a las personas su grado de satisfacción con diversos aspectos de su vida. Nuestro estudio se centra en el componente cognitivo del bienestar, constructo muy analizado en muestras de estudiantes y población general pero olvidado en otro tipo de colectivos como el de las personas con discapacidad intelectual (DI) (Dyckens, 2006). Los estudios sobre satisfacción vital en población con DI muestran, en relación a la satisfacción, un patrón similar que el encontrado en población general. Concretamente, los hallazgos muestran que la mayoría de las personas con DI suele evaluarse como más satisfechas que el promedio general en todas las áreas de la vida (Edgerton, 1996). Estos resultados suelen ser explicados en términos de bajas expectativas, poca elección o falta de experiencia (Raphael, Brown, Renwick y Rootman,
1996). Los escasos trabajos encontrados en este colectivo se han realizado con personas que han sufrido una experiencia traumática, que tienen una discapacidad física o aquéllas con DI severa, la mayoría de las cuales son incapaces de proporcionar una respuesta a preguntas relacionadas con el bienestar subjetivo (Perry y Felce, 2002, 2005; Silver, 1982). Hasta la fecha, la investigación interesada en analizar el bienestar en personas con DI ha sido relativamente escasa.

Con respecto a los predictores de los componentes del bienestar, diversos estudios han mostrado que la satisfacción vital está modulada por ciertos rasgos de personalidad (Judge, Erez, Bono y Thoresen, 2002; Law, Wong y Song, 2004). Por ejemplo, Judge et al. (2002) encontraron que la satisfacción vital estaba negativamente relacionada con neuroticismo en cuatro muestras de estudiantes. Así mismo, neuroticismo, extraversión, conciencia, apertura y agradabilidad estaban asociadas con la satisfacción con la vida en trabajadores (Law et al. 2004). Sin embargo hay que destacar, que de todos los rasgos anteriormente mencionados, neuroticismo y extraversión se han constatado como los predictores más importantes de la satisfacción vital (Diener, Oishi y Lucas, 2003).

Un constructo que ha generado un amplio campo de investigación en población sin discapacidad y que puede tener una potencial influencia sobre el bienestar subjetivo es el de las core self-evaluations (CSE) (He, Shi y Yi, 2014). El concepto de las CSE, que pudiera traducirse al castellano como autoevaluaciones centrales, fue desarrollado en la década de los 90 (Judge, Locke y Durham, 1997) para unificar una teoría global como explicación de resultados laborales complejos. Este concepto representa las evaluaciones fundamentales que los individuos mantienen sobre sí mismos, el mundo y los otros, las cuales a su vez influyen en las valoraciones de situaciones específicas, sin que en general seamos muy conscientes de esa influencia. Tales evaluaciones pueden ser positivas y negativas. En su formulación inicial del concepto los autores indican que las CSE son un rasgo amplio, latente y de orden superior compuesto por cuatro rasgos tradicionales en la literatura sobre personalidad: autoestima, autoeficacia generalizada, neuroticismo y locus de control. En lugar de considerar estas características de personalidad de manera independiente, las CSE plantean la consistencia de los distintos constructos de personalidad como un todo constituyendo así un factor global (Judge et al. 2002). Desde la psicología positiva, los investigadores están comenzando a evaluar la relación entre las CSE y el bienestar en población general (Baudin, Blanch, Rolland, Martí-Guiu y Aluja, 2014). Por ejemplo, Chang et al. (2012) en un metaanálisis realizado de 33 estudios encontraron que las CSE se relacionaba positiva y moderadamente con la satisfacción vital $(r=.54)$ en población sin discapacidad. Smedema et al. (2015) también hallaron una relación positiva entre las CSE y la satisfacción con la vida en el colectivo de personas con discapacidad, destacando la relevancia del constructo de CSE en esta población. 
Además, es necesario matizar que hasta la fecha, la mayoría de investigadores se han centrado en analizar la relación directa entre personalidad (neuroticismo, autoestima, etc... ) y satisfacción vital. Sin embargo, cada vez hay mayor evidencia de la influencia de mecanismos indirectos en la relación entre personalidad y satisfacción con la vida (He et al., 2014) y entre CSE y satisfacción vital en población sin discapacidad (Chang, Ferris, Johnson, Rosen y Tan, 2012). Este tipo de investigación interesada en descubrir los mecanismos subyacentes en la relación entre CSE y bienestar subjetivo en población sin discapacidad podría ser de vital importancia para tratar de identificar los posibles mediadores entre estas dos variables en personas con DI. Uno de esos mecanismos indirectos que podría mediar esa relación, sería el afecto positivo y negativo. Varios autores han encontrado que la relación entre afecto y satisfacción vital es compleja y que son constructos separables (Diener, Smith y Fujita, 1995; Lucas, Diener y Suh, 1996). Autores como Schimmack, (2008) han examinado la asociación entre la afectividad y la satisfacción vital en población sin discapacidad, encontrando que las personas suelen confiar en su balance afectivo para formarse juicios acerca de cómo de satisfechos están con sus vidas (Schwarz y Clore, 2007). De acuerdo con esto, investigaciones previas han confirmado una correlación positiva entre afecto positivo y satisfacción vital y una asociación negativa entre afecto negativo y satisfacción vital (Kuppens, Realo y Diener, 2008). Además, evidencias experimentales y transculturales han confirmado que la afectividad tiene una influencia causal sobre los juicios de satisfacción vital (Schwarz y Clore, 2007; Kuppens, Realo y Diener, 2008). Aunque más escasos, las evidencias en muestras con discapacidad encuentran una relación positiva entre el afecto positivo y la satisfacción vital y una relación negativa entre el afecto negativo y la satisfacción con la vida (van Leeuwen, Kraaijeveld, Lindeman y Post, 2012).

Por tanto, diversos estudios se han centrado en el papel mediador de la afectividad entre los rasgos de personalidad y la satisfacción vital proporcionando un acercamiento a los mecanismos subyacentes de esta relación (Liu, Wang y Li, 2012; Stoia-Caraballo et al. 2008). Sin embargo, son escasos los trabajos que han examinado el posible efecto mediador del afecto en la relación CSE y satisfacción vital en el colectivo de personas con discapacidad (Smedema et al., 2015), no encontrándose investigaciones que se centren exclusivamente en personas con DI. Por ello, el objetivo de esta investigación es analizar el posible efecto mediador de la afectividad positiva y negativa en la relación entre las CSE y la satisfacción vital en una muestra de personas con DI, lo que ayudaría a identificar posibles objetivos de intervención para posibilitar un mayor bienestar y calidad de vida en este colectivo.

\section{Participantes}

En el estudio participaron 153 personas con DI con una necesidad de apoyo intermitente o limitado, para sus activida- des de la vida diaria y residentes en la Comunidad Autónoma Andaluza, de los cuales 19 no completaron todos los cuestionarios o lo hicieron incorrectamente (marcando una única respuesta o un patrón determinado).

La muestra final estuvo formada por 134 personas con DI que acudían a entidades que trabajan en favor de este colectivo y participaban en un programa de orientación laboral (Andalucía Orienta). Las entidades participantes estuvieron distribuidas por 6 provincias de Andalucía: Málaga, Cádiz-Puerto Real, Almería, Granada, Jaén y Sevilla.

De los 134 sujetos, el 56.8\% eran varones, el 39.6\% eran mujeres. La edad oscilaba entre un mínimo de 20 años y un máximo de 52 años, observándose una media de 30.84 años (DT=6.28). En cuanto al estado civil el $61,2 \%$ de la muestra se encontraba sin pareja en el momento de cumplimentar los cuestionarios, estando casados o conviviendo en pareja el $5 \%$ de los sujetos. El resto de la muestra estaba soltera y con pareja o en otra situación diferente a las anteriores $(33,8 \%)$. En relación al nivel de formación, el $37.4 \%$ de los encuestados estaba en posesión del título de EGB o ESO, mientras que un $13.7 \%$ no tenía estudios o había realizado un programa cualificación profesional inicial.

\section{Instrumentos}

Core self-evaluations Scale (CSES, Judge, Bono y Thoresen, 2003).

Escala de 12 ítems desarrollada como medida directa de este constructo con una escala de respuesta que va desde 1=Fuertemente en desacuerdo/5 Fuertemente de acuerdo. Sus ítems, directos e inversos, permiten obtener una única puntuación que recoge los siguientes rasgos: autoestima, autoeficacia generalizada, neuroticismo y locus de control. La escala ha mostrado adecuadas propiedades psicométricas en diferentes estudios realizados con muestras españolas (Rey, Extremera y Durán, 2012; Rey, Extremera, Peláez-Fernández, 2016; Rey y Extremera, 2015). El índice de fiabilidad alpha obtenido en el estudio es de 0.81 .

Escala de satisfacción con la vida (SWLS; Diener, Emmons, Larsen y Griffin, 1985; adaptación española de Atienza, Pons, Balaguer y García-Merita, 2000).

Es un cuestionario de 5 ítems compuesto por una escala de tipo Likert de 7 puntos $(1=$ Completamente en desacuerdo/ $7=$ Completamente de acuerdo). Este instrumento es una medida global acerca del grado percibido de satisfacción en la vida. Diener et al. (1985) informaron de una elevada validez convergente y divergente y una alta consistencia interna $(\alpha=0.87)$. También Vassar (2008) en su meta-análisis apunta que la generalización de la fiabilidad de la escala tiene una consistencia interna de .78 con algún grado de variabilidad $(s=0.09)$. La validación al castellano de la escala presenta propiedades psicométricas similares a la versión anglosajona (Atienza et al., 2000). El valor de fiabilidad en este estudio fue satisfactorio $(\alpha=0.85)$. 
Escala de Afecto Positivo y Negativo (Positive Affect and Negative Affect; PANAS, Watson, Clark y Tellegen, 1988) versión española de Sandin et al., (1999).

Esta escala está compuesta por un listado de 20 adjetivos que describen sentimientos y emociones. La mitad de los adjetivos (10 ítems) evalúan afecto positivo, y la otra mitad (10 ítems) evalúan afecto negativo. Los participantes responden en una escala Likert de 5 opciones de acuerdo al grado en que las experimentan durante la última semana ( $1=$ Ligeramente $o$ nada/ 5=Mucho). Las escalas PANAS han demostrado ser una medida válida y fiable para evaluar la presencia y el grado del afecto positivo y negativo en población clínica y normal, de adolescentes, adultos y tercera edad (Robles y Paez, 2003). El índice de fiabilidad obtenido para cada una de las dimensiones son: afecto positivo $\alpha=.84$ y afecto negativo $\alpha=.86$.

\section{Procedimiento}

El primer paso de esta investigación fue el contacto con los gerentes de las entidades que trabajaban con personas con DI de Málaga y provincia para solicitar colaboración. Debido a las dificultades encontradas para acceder a todas las personas con DI susceptibles de participar en el estudio en la provincia de Málaga se solicitó la colaboración del programa de orientación laboral "Andalucía Orienta" presente en las distintas asociaciones de todas las provincias andaluzas. De las ocho provincias andaluzas, participaron Cádiz-Puerto Real, Almería, Granada, Jaén y Sevilla, siendo por tanto el tipo de muestro llevado a cabo en este estudio de tipo incidental.

La participación de las personas con DI fue totalmente voluntaria y anónima respetando la ley de protección de datos (Ley orgánica 15/1999 de 13 de diciembre) y las normas éticas correspondientes. Así mismo, los participantes firmaron un consentimiento informado de participación, dado que ninguno estaba incapacitado para ello y eran mayores de edad. La cumplimentación de la mayoría de los cuestionarios se hizo en la propia asociación de manera individual o grupal. En algunos casos en los que por motivos laborales, los participantes no podían acudir a la asociación para rellenarlos, se les proporcionaba para que los rellenaran en casa. Todos aquellos que lo rellenaron en las asociaciones y que lo necesitaron, recibieron un apoyo personal e individualizado para la comprensión de la terminología y el contenido de los ítems de mayor dificultad por parte de la investigadora principal. En los casos en los que los participantes los rellenaban en casa, en el momento de la entrega en la asociación, se revisaban con ellos aquellas cuestiones en las que tenían dudas y se les proporcionaba el apoyo necesario.

\section{Análisis de datos}

El análisis estadístico de los datos se llevó a cabo mediante el paquete estadístico para MAC SPSS, versión 24. En primer lugar se realizó el análisis de fiabilidad de cada una de las escalas de este estudio y se calcularon las medias, desviaciones típicas y coeficientes de correlación de Pearson. Posteriormente, para analizar el papel mediador del afecto, se realizaron análisis de mediación utilizando el procedimiento descrito por Preacher y Hayes (2008). Este procedimiento permite el análisis simultáneo y la comprobación estadística de cada uno de los efectos indirectos estimados (i.e. mediación) en un modelo a través de cada mediador y los efectos directos de la variable predictora (i.e. CSE) sobre la variable dependiente (i.e. satisfacción vital), controlando los efectos potenciales de otras posibles variables. En este caso caso, para evitar posibles efectos de covariables, se controló en los análisis la edad y el sexo. Se utilizó el procedimiento bootstrapping con 10000 repeticiones ya que tiene mayor control del error Tipo I que otros análisis de mediación y determina si el efecto indirecto de los mediadores es significativamente diferente de cero a través de los intervalos de confianza del $95 \%$. Si para cada efecto indirecto, el intervalo de confianza no incluye el 0 , entonces el efecto indirecto es significativo $(\mathrm{p}<.05) \mathrm{y}$, por tanto, se puede afirmar que la mediación existe. Para ello se utilizó la macro de SPSS proporcionada por Preacher y Hayes (2008).

\section{Resultados \\ Análisis descriptivos}

En la Tabla 1 aparecen las correlaciones, medias, desviaciones típicas y fiabilidades de las variables utilizadas en este estudio.

Tabla 1

Medias, desviaciones típicas, fiabilidades y correlaciones entre las medidas evaluadas.

\begin{tabular}{lcccccccc}
\hline & M & S.D. & $\alpha$ & 1 & 2 & 3 & 4 & 5 \\
\hline 1. CSE & 3,47 &, 55 & .81 & - & & & & \\
2. Afecto positivo & 3,81 &, 78 & .84 & $.54^{* *}$ & - & & & \\
3. Afecto negativo & 2,12 &, 86 & .86 & $-.54^{* *}$ &.$-.21^{*}$ & - & & \\
4. Satisfacción vital & 5,30 & 1,28 & .85 & $.40^{* *}$ & $.44^{* *}$ &.-.11 & - & \\
\hline
\end{tabular}

Nota: ${ }^{*} p<0.05 ; * * p<0.01$

Como se puede apreciar en la Tabla 1, las CSE se relacionaron negativa y positivamente, según el caso, con las dos dimensiones del afecto (afecto positivo, $r=.54, p<.01$; afecto negativo, $r=-.54, p<.01)$ así como con la satisfacción vital $(r=.40, p<.01)$. Por otro lado, tal como era esperable, el afecto positivo se relacionó con satisfacción vital de modo positivo y significativo $(r=.44, p<.01)$. Sin embargo, el afecto negativo no correlacionó de forma estadísticamente significativa con satisfacción vital $(r$ $=-.11, p>.05)$.

\section{Análisis mediacionales}

Para examinar el papel mediador de las dimensiones del afecto en la relación entre CSE y satisfacción vital, se utilizó la macro de SPSS proporcionada por Preacher y Hayes (2008). Utilizando dicha macro para realizar análisis de mediaciones múltiples con dos mediadores (afecto positivo y afecto negativo) y controlando el efecto de dos covariables (edad y sexo) encontramos los resultados que aparecen en la Tabla 2. 
Tabla 2

Análisis de los efectos mediacionales múltiples del afecto sobre la relación entre Core self-Evaluations y satisfacción vital mediante el método bootstrapping.

\begin{tabular}{|c|c|c|c|c|c|c|c|c|}
\hline $\begin{array}{c}\text { Variable } \\
\text { Independiente } \\
\text { (VI) }\end{array}$ & Mediadores & $\begin{array}{c}\text { Variable } \\
\text { dependiente } \\
\text { (VD) }\end{array}$ & $\begin{array}{c}\text { Efecto de } \\
\text { la VI a los } \\
\text { mediadores (a) }\end{array}$ & $\begin{array}{l}\text { Efecto directo de } \\
\text { los mediadores a } \\
\text { la VD (b) }\end{array}$ & $\begin{array}{l}\text { Efecto Total } \\
\text { (c) }\end{array}$ & $\begin{array}{l}\text { Efecto Directo } \\
\text { De la VI sobre la } \\
\text { VD (c') }\end{array}$ & $\begin{array}{c}\text { Efecto de las } \\
\text { covariables } \\
\text { sobre VD }\end{array}$ & $\begin{array}{c}\text { Intervalo de } \\
\text { Confianza (IC) } 95 \% \\
\text { (inferior y superior) }\end{array}$ \\
\hline \multirow[t]{3}{*}{ CSE } & & $\begin{array}{c}\text { Satisfacción } \\
\text { vital }\end{array}$ & & & $.99 * *$ & $.81 * *$ & $\begin{array}{l}\text { Sexo: } 0.02(\mathrm{~ns}) \\
\text { Edad: } 0.01(\mathrm{~ns})\end{array}$ & \\
\hline & $\begin{array}{c}\text { Afecto } \\
\text { positivo }\end{array}$ & & $.75^{* *}$ & $.49^{* *}$ & & & & $0.110-0.690$ \\
\hline & $\begin{array}{c}\text { Afecto } \\
\text { negativo }\end{array}$ & & $-.86^{* *}$ & .22 & & & & $-0.443--0.022$ \\
\hline
\end{tabular}

Nota: $* * p<0.01$

Ninguna de las covariables introducidas en el modelo final, mostró su influencia significativa en la relación entre CSE y satisfacción vital. El intervalo de confianza del modelo cuando se analizó la variable mediadora de afecto positivo se encontraba entre 0.110 y 0.690 con un intervalo de confianza del 95\%. Esto mostró evidencias significativas de mediación puesto que no se encontraba el cero entre ese intervalo. Los hallazgos sugieren que el efecto indirecto fue significativo $(p<.05)$, indicando la presencia de mediación de afecto positivo sobre la relación entre CSE y satisfacción vital. Por otro lado, el efecto indirecto del afecto negativo se encontraba entre -.443 y -.022 con un intervalo de confianza del $95 \%$. Al estar el cero en ese intervalo se sugiere que no existe mediación sobre la relación entre CSE y satisfacción vital.

Es necesario subrayar que el afecto positivo opera como mediador en la relación entre CSE y satisfacción vital actuando como mediador parcial ya que el efecto directo de las CSE sobre satisfacción vital continuaba siendo estadísticamente significativo $\left(c^{\prime}=0.81 ; p<.00\right)$. El modelo final obtenido aparece en la Figura 1.

\section{Figura 1}

Modelo de mediación múltiple: la variable independiente $\mathrm{X}$ influye directamente sobre la variable dependiente $\mathrm{Y}$ ( $\left.\mathrm{c}^{\prime}\right)$ o indirectamente vía M1 y M2 ( $a, b$ y c). La influencia de las dimensiones edad y sexo (C) son controladas en la relación.

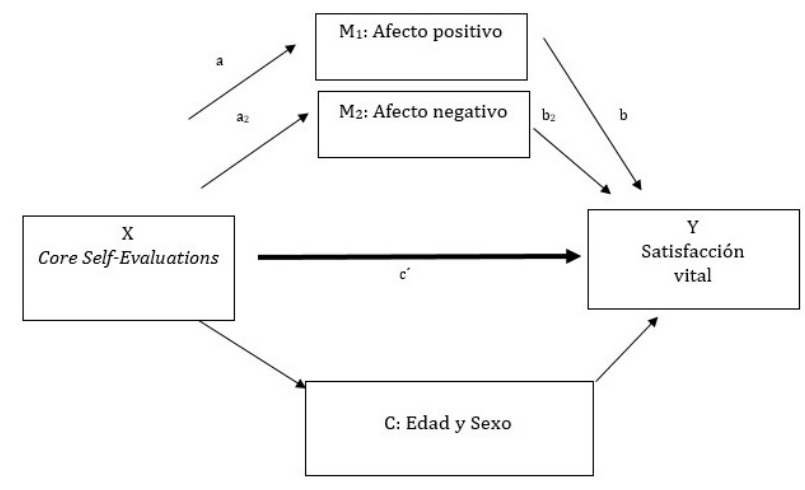

\section{Discusión}

El presente trabajo examina el papel mediador del afecto en la relación CSE y satisfacción vital en una muestra de personas con DI. Estos hallazgos podrían aportar evidencias para identificar posibles objetivos de intervención que posibiliten un mayor bienestar y calidad de vida en este colectivo.

Consistente con otros estudios que analizan la relación entre personalidad y bienestar en diferentes muestras (DeNeve y Cooper, 1998; Heller, Watson e Ilies, 2004), el presente trabajo encontró que las CSE son importantes predictores del bienestar en personas con DI. Estos hallazgos sugieren evidencias de que determinados rasgos de personalidad considerados en su conjunto y que constituyen las CSE, son importantes predictores de la satisfacción vital en personas con DI. Concretamente los resultados mostraron que los niveles de CSE se asociaban a mayores puntuaciones en satisfacción vital, por lo que nuestros resultados parecen mostrar evidencias de que las CSE pueden ser otro recurso personal relevante a tener en cuenta junto con el listado de constructos ya clásicamente estudiados. Diversos autores ya habían apuntado previamente la importancia del uso de las CSE para comprender el bienestar en población general (Judge, Erez, Bono, y Thoresen, 2002) y en estudiantes con diferentes discapacidades (Smedema, et al., 2015). Nuestros hallazgos sugieren, en línea con estos estudios, que las evaluaciones globales que hacen las personas con DI sobre ellas mismas son más significativas para comprender su satisfacción con la vida.

Igualmente, encontramos que las personas con DI con mayor CSE también indicaban mayores niveles de afecto positivo y menores niveles de afecto negativo. Estos resultados confirman evidencias previas de que las CSE correlacionan positivamente con mayores niveles de afecto en población con discapacidad (van Leeuwen et al., 2012). En una revisión sistemática previa realizada en personas con lesión medular, los autores encuentran una relación positiva entre CSE y afecto positivo y una relación negativa entre CSE y afecto negativo (van Leeuwen et al., 2012). De igual forma nuestro estudio constata que las personas con DI que tienen altas CSE informaban de un mayor afecto positivo y un menor afecto negativo, lo que redundaba en mayores niveles de satisfacción con la vida.

Por otro lado, los resultados de los análisis mediacionales múltiples proporcionaron información sobre cómo las CSE inciden sobre la satisfacción vital. Concretamente los resultados indicaron que las CSE tenían una influencia directa sobre la satisfacción vital e indirecta, vía afecto positivo. De acuerdo con el modelo, las personas con DI con altas CSE están más 
satisfechas con sus vidas porque experimentan mayores niveles de afecto positivo. Estos hallazgos son consistentes con el patrón obtenido en estudios previos realizados con adolescentes (He et al., 2014) y con estudiantes con diferentes discapacidades (Smedema et al., 2015) donde se encontraron que el afecto mediaba la relación entre las CSE y la satisfacción vital.

Nuestros hallazgos pueden ser de interés no sólo para ampliar la propuesta sobre recursos personales como predictores de la satisfacción vital en el colectivo de personas con DI, sino también, para poder diseñar y desarrollar programas de intervención dirigidos a fomentar el bienestar en esta muestra. La DI es un concepto dinámico y activo, una característica más de la persona y no una enfermedad. Por este motivo, las personas con DI evolucionan y son capaces de integrarse satisfactoriamente en la comunidad. Este es uno de los valores que guían a las organizaciones que trabajan cada día por mejorar la calidad de vida de este colectivo. La planificación de programas y actuaciones que fomentan el desarrollo personal pleno, podría beneficiarse del conocimiento de que las CSE se asocian con la satisfacción con la vida así como el conocimiento de qué otras variables están influyendo, aumentando o disminuyendo el efecto de estos recursos sobre el bienestar.

Por tanto, en términos de intervención, los programas dirigidos a fomentar la calidad de vida en personas con DI deberían integrar elementos para fomentar una buena autoestima, una baja estabilidad emocional, una alta autoeficacia generalizada y un locus de control adecuado a la situación. En definitiva, una formación orientada a aumentar los recursos personales, podría aumentar las experiencias emocionales positivas lo que redundaría en una mayor satisfacción con la vida en el colectivo de personas con DI.

Por otro lado, y en consonancia con Judge y KammeyerMueller (2008), los resultados de este estudio proporcionan evidencias de la importancia del afecto como mediador entre las CSE y la satisfacción vital. Este estudio pone de manifiesto que el afecto como estado es una variable predictora de la satisfacción vital. Tal y como sugiere Brief (1998), nuestros resultados parecen apoyar la idea de que el afecto y las CSE son conceptos que hacen contribuciones diferentes sobre la satisfacción vital, puesto que la mediación encontrada no es total sino parcial y sugieren diferentes vías para intervenir en programas psicosociales para la mejora de los niveles de satisfacción con la vida.

A pesar de las evidencias preliminares encontradas, los resultados deben ser interpretados con cautela debido a diversas limitaciones. Una de ellas, hace referencia a la relación entre los recursos personales y las dimensiones de bienestar personal. Al tratarse de un estudio de carácter transversal la relación causal no es posible. No obstante, el planteamiento del modelo mediacional y el orden de las variables está basado en evidencias empíricas anteriores (Kuppens, Realo y Diener, 2008, Schwarz y Clore, 2007). Al igual que en otros ámbitos de investigación sería de gran interés el desarrollo de estudios longitudinales que pudieran analizar empíricamente las posibles relaciones cau- sales entre los recursos personales y el bienestar. Además, es importante subrayar que la selección de la muestra está basada en un método de selección de conveniencia o muestreo incidental, puesto que todos los participantes acudían a un servicio de orientación laboral para personas con DI. Por otro lado, todos los participantes del estudio eran personas con con una necesidad de apoyo intermitente o limitado para sus actividades de la vida diaria, por lo que los resultados pueden no ser generalizables a personas con DI con otras necesidades e intensidades de apoyo. Futuros trabajos deberían aplicar diseños aleatorios de selección de la muestra y considerar diferentes necesidades e intensidades de apoyo. Por último, este estudio utiliza en exclusiva medidas de autoinforme. Este método puede tener sesgos en términos de deseabilidad social y autoengaño. En este sentido sería útil incluir otros métodos de evaluación basado en entrevistas a observadores.

A pesar de las limitaciones, el presente estudio sugiere que las CSE están asociadas a una mejor satisfacción vital en las personas con DI, intermediando en esa relación el afecto de los individuos. Nuestros resultados pueden tener implicaciones para la práctica clínica y la formación de personas con DI orientada a aumentar los recursos personales para favorecer un mayor bienestar emocional potenciando con ello la calidad de vida, tan importante en nuestra sociedad del bienestar.

\section{Agradecimientos}

La presente investigación pudo llevarse a cabo gracias a la colaboración de las siguientes asociaciones de la Comunidad Autónoma Andaluza: AMADPSI, APM-IBERIA, ADIPA, ASPROMANIS, ASPANDEM, SÍNDROME DE DOWN, FAHALA, FEPROAMI, ASPRODESA, FUNDACIÓN DOCETE OMNES, APROMPSI Y APROSE. Igualmente queremos agradecer la colaboración de todas las personas con DI que tomó parte en el estudio.

\section{Referencias}

1. Atienza, F.L., Pons, D, Balaguer, I y García-Merita, M (2000). Propiedades psicométricas de la escala de satisfacción con la vida en adolescentes. Psicothema 12, 314-319.

2. Baron, R. M. y Kenny, D. A. (1986). The moderator-mediator variable distinction in social psychological research: Conceptual, strategic and statistical considerations. Journal of Personality and Social Psychology, 51, 1173-1182. https://doi.org/10.1037/0022-3514.51.6.1173

3. Baudin, N., Blanch, A., Rolland, J.P., Martí-Guiu, M. y Aluja, A. (2014). Core Self-Evaluations, life satisfaction, and sport satisfaction. Escritos de Psicología, 3, 19-24. https://doi.org/10.5231/psy.writ.2014.2507

4. Brief, A.P. (1998). Attitudes In and Around Organizations. Thousand Oaks, CA: Sage.

5. Chang, C.-H., Ferris, D. L., Johnson, R. E., Rosen, C. C., y Tan, J. A. (2012). Core self evaluations: A review and evaluation of the literature. Journal of Management, 38, 
81-128. https://doi.org/10.1177/0149206311419661

6. Danna, K. y Griffin, R. (1999). Health and well-being in the workplace: A review and synthesis of the literature. Journal of Management, 25, 357-384. https://doi. org/10.1177/014920639902500305

7. DeNeve, K. M. y Cooper, H. (1998). The happy personality: A meta-analysis of 137 personality traits and subjective well-being. Psychological Bulletin, 124, 197-229. https:// doi.org/10.1037/0033-2909.124.2.197

8. Diener, E., Emmons, R. A., Larsen, R. J. y Griffin, S. (1985). The satisfaction with life scale. Journal of Personality Assessment, 49, 71-75. https://doi.org/10.1207/ s15327752jpa4901_13

9. Diener, E., y Lucas, R. E. (1999). Personality and subjective well-being. In D. Kahneman, E. Diener, y N. Schwarz (Eds.), Well-being: The foundations of hedonic psychology (pp. 213-229). New York: Russell Sage Foundation.

10. Diener, E., Oishi, S., y Lucas, R. E. (2003). Personality, culture, and subjective well being: Emotional and cognitive evaluations of life. Annual Review of Psychology, 54, 403-425. https://doi.org/10.1146/annurev. psych.54.101601.145056

11. Diener, E., Sandvik, E. y Pavo t, W. (1991). Happiness is the frequency, not the intensity, of positive versus negative affect. En F. Strack, M. Argyle y N. Schwarz (Eds.), Subjective well-being: An interdisciplinary perspective (pp. 119139). New York: Pergamon.

12. Diener, E., Scollon, C. N. y Lucas, R. E. (2003). The evolving concept of subjective well-being: The multifaceted nature of happiness. In P. T. Costa y I. C. Siegler (Eds.), Advances in cell aging and gerontology: Vol. 15 (pp. 187220). Amsterdam: Elsevier. https://doi.org/10.1016/s15663124(03)15007-9

13. Diener, E., Smith, H. y Fujita, F. (1995). The personality structure of affect. Journal of Personality and Social Psychology, 69, 130-141. https://doi.org/10.1037/00223514.69.1.130

14. Diener, E., Suh, E. M., Lucas, R. E. y Smith, H. L. (1999). Subjective well-being: Three decades of progress. Psychological Bulletin, 125, 276-302. https://doi.org/10.1037/00332909.125.2.276

15. Dykens, E.M. (2006). Toward a positive psychology of mental retardation. American Journal of Orthopsychiatry, 76(2), 185-193. https://doi.org/10.1037/0002$\underline{9432.76 .2 .185}$

16. Edgerton, R.B. (1996). A longitudinal-ethnographic research perspective on quality of life. In R. L. Schalock (Ed.), Quality of life: Volume I: Conceptualization and measurement (pp. 83-90). Washington, DC: American Association on Mental Retardation.

17. He, D., Shi, M. $y$ Yi, F. (2014). The mediating effects of affect and loneliness on the relationship between core self-evaluation and life satisfaction among two groups of
Chinese adolescents. Social Indicators Research, 119, 747756. https://doi.org/10.1007/s11205-013-0508-3

18. Heller, D., Watson, D. y Ilies, R. (2004). The role of person versus situation in life satisfaction: A critical examination. Psychological Bulletin, 130, 574-600. https://doi. org/10.1037/0033-2909.130.4.574

19. Judge, T. A., Erez, A., Bono, J. E., y Thoresen, C. J. (2002). Are measures of self-esteem, neuroticism, locus of control, and generalized self-efficacy indicators of a common core construct? Journal of Personality and Social Psychology, 83, 693-710. https://doi.org/10.1037/0022-3514.83.3.693

20. Judge, T. A., Erez, A., Bono, J. E., y Thoresen, C. J. (2003). The Core Self-Evaluations Scale (CSES): Development of a measure. Personnel Psychology, 56, 303-331. https://doi. org/10.1111/j.1744-6570.2003.tb00152.x

21. Judge, T.A., Erez, A., Bono, J.E. y Thoresen, C.J. (2002). Are measures of self-esteem, neuroticism, locus of control, and generalized self-efficacy indicators of a common core construct? Journal of Personality and Social Psychology, 83, 693-710. https://doi.org/10.1037/0022-3514.83.3.693

22. Judge, T.A., Locke, E.A. y Durham, C.C. (1997). The dispositional causes of job satisfaction: A core evaluations approach. Research in Organizational Behavior, 19, 15188.

23. Kahneman, D., Diener, E. y Schwarz, N. (1999). Wellbeing: the foundations of hedonic psychology. New York: Russell- Sage.

24. Kuppens, P., Realo, A., y Diener, E. (2008). The role of positive and negative emotions in life satisfaction judgment across nations. Journal of Personality and Social Psychology, 95, 66-75. https://doi.org/10.1037/0022-3514.95.1.66

25. Larsen, R. J., Diener, E. y Emmons, R.A. (1985). An evaluation of subjective well-being measures. Social Indicators Research, 17, 1-18. https://doi.org/10.1007/BF00354108

26. Law, K. S., Wong, C. S., y Song, L. J. (2004). The construct and criterion validity of meta-mood experience and its potential utility for management studies. Journal of Applied Psychology, 89, 483-496. https://doi.org/10.1037/0021$\underline{9010.89 .3 .483}$

27. Liu, Y., Wang, Z. H., y Li, Z. G. (2012). Affective mediators of the influence of neuroticism and resilience on life satisfaction. Personality and Individual Difference, 52, 833-838. https://doi.org/10.1016/j.paid.2012.01.017

28. Lucas, R. E., Diener, E. y Suh, E. (1996). Discriminant validity of well-being measures. Journal of Personality and Social Psychology, 71, 616-628. https://doi. org/10.1037/0022-3514.71.3.616

29. Perry J, Felce D. (2002) Subjective and objective quality of life assessment: Responsiveness, response bias and resident:proxy concordance. Mental Retardation 40, 445-456. https://doi.org/10.1352/00476765(2002)040<0445:SAOQOL $>2.0 . \mathrm{CO} ; 2$

30. Perry, J. y Felce, D. (2005). Correlation between sub- 
jective and objective measures of outcome in staffed community housing. Journal of Intellectual Disability Research, 49, 278-287. https://doi.org/10.1111/j.13652788.2005.00652.x

31. Preacher, K. y Hayes, A. (2008) Asymptotic and resampling strategies for assessing and comparing indirect effects in multiple mediator models. Behavior Research Methods 40, 879-891. https://doi.org/10.3758/BRM.40.3.879

32. Raphael D., Brown I., Renwick R. y Rootman I. (1996). Assessing the quality of life of persons with developmental disabilities: description of a model, measuring instruments, and initial findings. International Journal of Disability, Development and Education 43, 25-42. https:// doi.org/10.1080/0156655960430103

33. Rey, L., Extremera, N y Durán. M.A (2012). Core selfevaluations, meta-mood experience, and happiness: Tests of direct and moderating effects. Personality and Individual Differences 53, 207-212. https://doi.org/10.1016/j. paid.2012.03.009

34. Rey, L. y Extremera, N. y Peláez-Fernández, M.A. (2016). Linking Social Support to Psychological Distress in the Unemployed: The Moderating Role of Core Self-Evaluations. Social Indicators Research 127, 435-445. https://doi. org/10.1007/s11205-015-0958-x

35. Rey, L. y Extremera, N. (2015). Core Self-Evaluations, Perceived Stress and Life Satisfaction in Spanish Young and Middle-Aged Adults: An Examination of Mediation and Moderation Effects. Social Indicators Research 120, 515-524. https://doi.org/10.1007/s11205-014-0601-2

36. Robles, R. y Paéz, F. (2003). Estudio sobre la traducción al español y las propiedades psicométricas de las escalas de afecto positivo y negativo (PANAS). Salud Mental, 26, 69-75.

37. Russell, J. E. A. (2008). Promoting subjective well-being at work. Journal of Career Assessment, 16, 117-131. https:// doi.org/10.1177/1069072707308142

38. Schimmack, U. (2008). The structure of subjective wellbeing. In Eid,M. and Larsen,R. J. (Eds.), The Science of subjective well-being (pp. 97-123). New York: The Guilford Press.

39. Schwarz, N., y Clore, G. L. (2007). Feelings and phenomenal experiences. In A. Kruglanski y E. T. Higgins (Eds.), Social psychology. Handbook of basic principles (2nd ed., pp. 385-407). New York: Guilford Press.
40. Seligman, M. y Csikszentmihalyi, M. (2000). Positive psychology: an introduction. American Psychologist, 55, 5-14. https://doi.org/10.1037/0003-066X.55.1.5

41. Shin, D, C. y Johnson, D.M. (1978). Avowed happiness as an overall assessment of the quality of life. Social Indicators Research, 5, 475-492. https://doi.org/10.1007/BF00352944

42. Silver, R.L. (1982). Coping with and undesirable life event: a study of early reations to physical disability. Unpublished doctoral dissertation, Noorthwestern Universtiy, Evanston, Illionois.

43. Smedema, S; Chan, F; Yaghmaian, R; Cardoso, E; Muller, V; Keegan, J; Dutta, A y Ebener, D. (2015). The relationship of core self-evaluations and life satisfaction in college students with disabilities: evaluation of a mediator model. Journal of Postsecondary Education and Disability, 28, 341-358

44. Stoia-Caraballo, R., Rye, M. S., Pan, W., Kirschman, K. J. B., Lutz-Zois, C., y Lyons, A. M. (2008). Negative affect and anger rumination as mediators between forgiveness and sleep quality. Journal of Behavioral Medicine, 31, 478-488. https://doi.org/10.1007/s10865-008-9172-5

45. van Leeuwen CM, Kraaijeveld S, Lindeman E, Post MW. (2012). Associations between psychological factors and quality of life ratings in persons with spinal cord injury: a systematic review. Spinal cord 50, 174-87. https://doi. org/10.1038/sc.2011.120

46. Vassar, M. (2008). A note on the score reliability for the Satisfaction With Life Scale: an RG study. Social Indicators Research, 86, 47-57. https://doi.org/10.1007/s11205-0079113-7

47. Vázquez, C. y Hervás, G. (2009). La ciencia del bienestar: fundamentos de una psicología positiva. Madrid: Alianza Editorial.

48. Watson, D., Clark, L. A. y Tellegen, A. (1988). Development and validation of brief measures of positive and negative affect: The PANAS scale. Journal of Personality and Social Psychology, 54, 1063-1070. https://doi.org/10.1037/0022$\underline{3514.54 .6 .1063}$

RECIBIDO: 08/06/2016

MODIFICADO: 23/08/2016

ACEPTADO: $12 / 12 / 2016$ 